\title{
Molecular detection and sero-prevallence of Leishmania donovani risk factor analysis in selected areas in Sri Lanka.
}

by

Peduru Hewa Kankanange Imalka Shalindra Ranasinghe

Thesis subminitted to the Universituty of Sri Jayewardemeptura for the awand off the Degneee off Donctooroff PHhilbsepphyoonl I5 of August 2013 


\section{Declaration by the Candidate}

I declare that the work described in this thesis was carried out by me under the supervision of Professor Renu Wickremasinghe, Head, Department of Parasitology, Faculty of Medical Sciences, University of Sri Jayewardenepura, Dr. NV Chandrasekharan, Department of Chemistry, University of Colombo, Professor Nissanka De Silva, Head, Department of Zoology, University of Sri Jayewardenepura, Dr. Rhayza Maingon, Senior Lecturer, Centre for Applied Entomology and Parasitology, ISTM, Keele University, ST5 5BG, Staffordshire, UK and Professor Greg Matlashewski, Chairman, Department of Microbiology and Immunology, McGill University, Montreal, Canada; Special Program for Research and Training in Tropical Diseases, World Health Organization, Switzerland and a report on this has not been submitted in whole or in part to any university or any other institution for another Degree/Diploma".

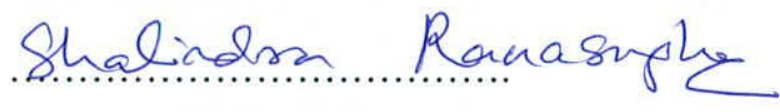

Dr. PHKI Shalindra Ranasinghe

$$
15.07 .2014
$$

Date

We certify that the candidate has incorporated all possible corrections and amendments recommended by the examiners.

P

Professor Renu Wickremasinghe, $1517 / 2014$

Principal Supervisor/Head,

Department of Parasitology,

Faculty of Medical Sciences,

University of Sri Jayewardenepura

M. Chendronetharac

Dr. NV Chandrasekharan,

Supervisor/Senior Lecturer,

Department of Chemistry,

University of Colombo

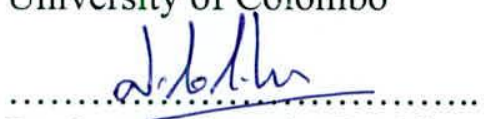

Professor Nissanka De Silva,

Supervisor/Head,

Department of Zoology,

University of Sri Jayewardenepura

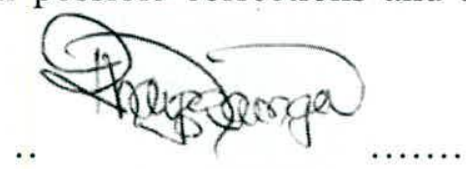

Dr. Rhayza Maingon, Supervisor/Senior Lecturer, Centre for Applied Entomology and Parasitology,

ISTM, Keele University, ST5 5BG,

Staffordshire, UK

Professor Greg Matlashewski,

Supervisor/Chairman, Department of Microbiology and Immunology,

McGill University, Montreal, Canada;

Special Program for Research and Training in Tropical Diseases, World Health Organization,

Switzerland 


\section{Declaration by the Candidate}

I declare that the work described in this thesis was carried out by me under the supervision of Professor Renu Wickremasinghe, Head, Department of Parasitology, Faculty of Medical Sciences, University of Sri Jayewardenepura, Dr. NV Chandrasekharan, Department of Chemistry, University of Colombo, Professor Nissanka De Silva, Head, Department of Zoology, University of Sri Jayewardenepura, Dr. Rhayza Maingon. Senior Lecturer, Centre for Applied Entomology and Parasitology, ISTM, Keele University, ST5 5BG, Staffordshire, UK and Professor Greg Matlashewski, Chairman, Department of Microbiology and Immunology, McGill University, Montreal, Canada; Special Program for Research and Training in Tropical Diseases, World Health Organization, Switzerland and a report on this has not been submitted in whole or in part to any university or any other institution for another Degree/Diploma".

$$
\text { Shalindra Ranasighe } \quad 15.07 .2014
$$

Dr. PHKI Shalindra Ranasinghe

Date

We certify that the candidate has incorporated all possible corrections and amendments recommended by the examiners.

Renu hiecunasing

Professor Renu Wickremasinghe, Principal Supervisor/Head,

Department of Parasitology,

Faculty of Medical Sciences,

University of Sri Jayewardenepura

MN Chemdrathouar

Supervisor/Senior Lecturer,

Department of Chemistry,

University of Colombo

Professor Nissanka De Silva,

Supervisor/Head,

Department of Zoology,

University of Sri Jayewardenepura

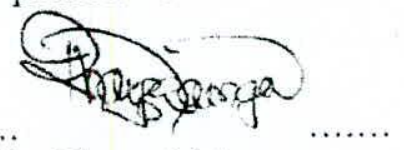

Dr. Rhayza Maingon,

Supervisor/Senior Lecturer,

Centre for Applied Entomology

and Parasitology,

ISTM, Keele University,

ST5 5BG,

Staffordshire,

UK

Professor Greg Matlashewski,

Supervisor/Chairman, Department of

Microbiology and Immunology,

McGill University,

Montreal, Canada;

Special Program for Research and

Training in Tropical Diseases,

World Health Organization,

Switzerland 


\section{Declaration by the supervisors}

We certify that the above statement made by the candidate is true and that this thesis is suitable for submission to the University for the purpose of evaluation.

Renn hichanasfur $1517 / 2014$

Professor Renu Wickremasinghe,

Head, Department of Parasitology,

Faculty of Medical Sciences,

University of Sri Jayewardenepura

M. Chandianlharas

Dr. NV Chandrasekharan, ioj) July/20\%

Senior Lecturer,

Department of Chemistry,

University of Colombo

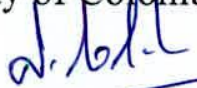

Professor Nissanka De Silva,

Head, Department of Zoology,

University of Sri Jayewardenepura

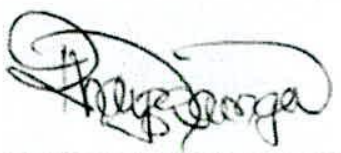

Dr. Rhayza Maingon,

Senior Lecturer,

Centre for Applied Entomology and Parasitology,

ISTM, Keele University,

ST5 5BG,

Staffordshire,

UK

Professor Greg Matlashewski,

Chairman, Department of Microbiology and Immunology,

McGill University,

Montreal, Canada;

Special Program for Research and Training in Tropical Diseases,

World Health Organization,

Switzerland 


\section{Declaration by the supervisors}

We certify that the above statement made by the candidate is true and that this thesis is suitable for submission to the University for the purpose of evaluation.

R

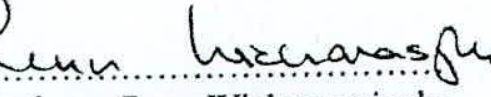

Professor Renu Wickremasinghe,

Head, Department of Parasitology,

Faculty of Medical Sciences,

University of Sri Jayewardenepura

M Chandracthouar.

Dr. NV Chandrasekharan, isjuinglzous

Senior Lecturer,

Department of Chemistry,

University of Colombo

Professor Nissanka De Silva,

Head, Department of Zoology,

University of Sri Jayewardenepura

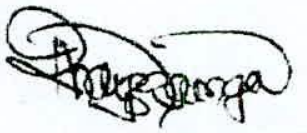

Dr. Rhayza Maingon,

Senior Lecturer,

Centre for Applied Entomology and Parasitology,

ISTM, Keele University,

ST5 5BG,

Staffordshire,

UK

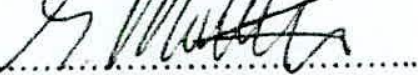

Professor Greg Matlashewski,

Chairman, Department of Microbiology and Immunology,

McGill University,

Montreal, Canada;

Special Program for Research and Training in Tropical Diseases,

World Health Organization,

Switzerland 
The work given below, described in this Thesis was kindly carried out by the following collaborators since the necessary facilities were not available in Sri Lanka and the expertise was not available within the Department.

1. Multi Locus Enzyme Electrophoresis (MLEE) of the endogenous visceral leishmanisis causing strain in Sri Lanka was carried out kindly by Dr. Francine Pratlong and Laboratoire de Parasitologie-Mycologie, CHRU de Montpellier, Centre National de Référence des Leishmania, UMR MIVEGEC (Université Montpellier 1-CNRS 5290-IRD 224), Montpellier, France.

2. 6-Phogluconated dehydrogenase (6PGDH) partial gene sequencing of CL \& VL strains was kinldy carried out by Professor Greg Matlashewski and Dr. Wen-wei Zhang, of McGill University, Canada.

3. Principal Componant Analysis (PCA) was kindly carried out by Dr. Daniel Bray of Keele University, UK.

4. Global Positioning System (GPS) distance calculations and mapping was kindly carried out by Mr. Sundaramoorthy Sivanantharajah, GIS Branch, Department of Surveys, Sri Lanka 


\section{Contents}

page

Contents

List of Tables vii

List of Figures

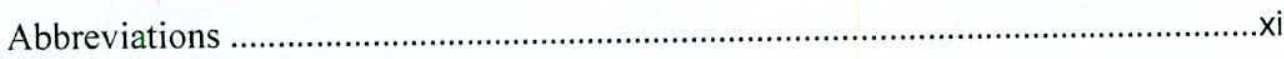

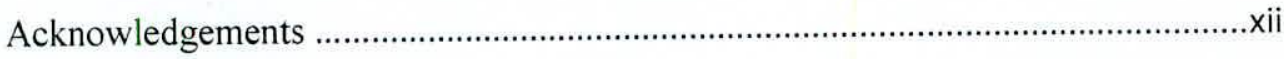

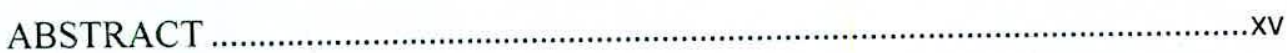

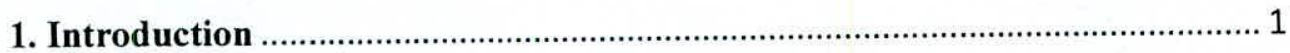



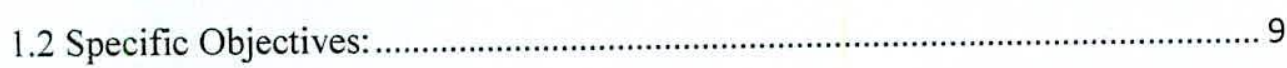

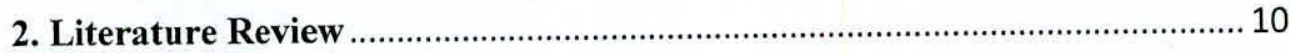

3. Materials and Methods ............................................................................. 15

3.1 Isolation of visceral and cutaneous leishmaniasis causing strains in Sri Lanka 15

3.1.1. Culture of the VL strain and submission for strain identification ................. 15

3.1.2 Culture of the CL strain .......................................................................... 17

3.1.3 Multi Locus Enzyme Electrophoresis (MLEE) …......................................... 17

3.1.4 Sequencing of 6-Phospho gluconate dehydrogenase gene (6PGDH)............. 18

3.2. A cross sectional study to assess the sero- prevalence and risk factors for leishmaniasis in an endemic region of Sri Lanka ........................................... 19






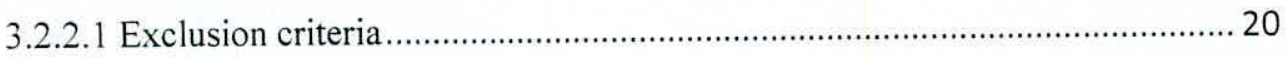

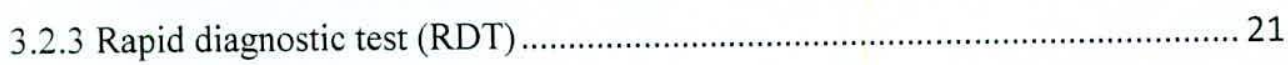

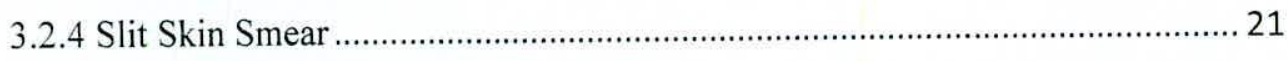

3.2.5 Socio-demographic data collection............................................................. 22

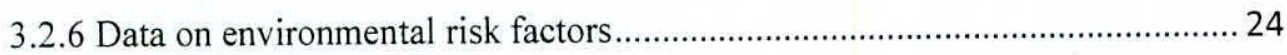

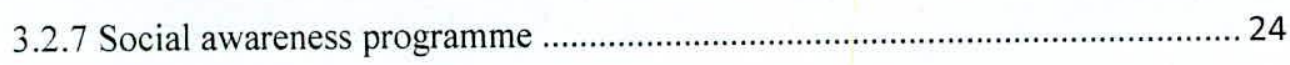

3.2.8 Management and follow up of newly diagnosed leishmaniasis patients ........ 24



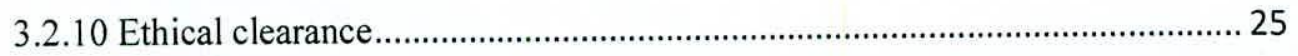

3.3 Detection of Leishmania donovani DNA using DNA based methods in Sri Lanka 26

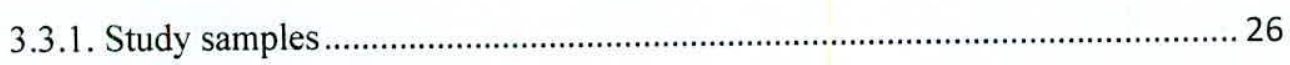

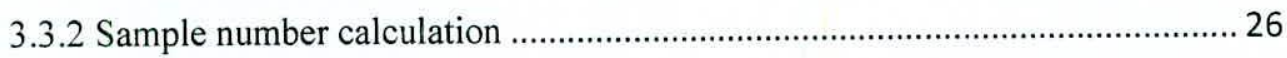

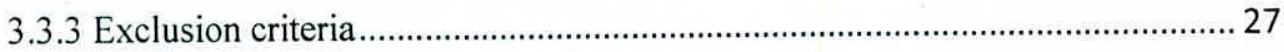

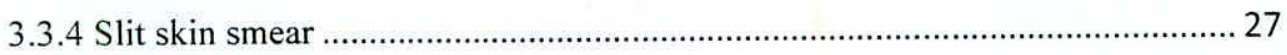



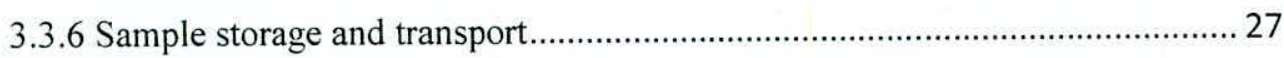

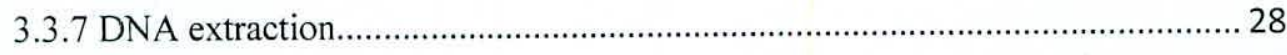

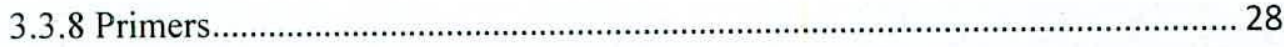

3.3.8.1 Genus Leishmania specific Kinetoplast (kDNA) PCR ................................ 29

3.3.8.2 Leishmania donovani species specific PCR …......................................... 30

3.3.8.3 Internal Transcribed Spacer I (ITS 1) PCR ….......................................... 31 
3.3.10 Positive controls

3.3.11 Negative controls

3.3.12 PCR product visualization and capture.

3.3.13 Ethical clearance. 34

3.4 Taxonomic identification of sand flies captured from diagnosed CL patients' habitats in Matale district; an active transmission area for $\mathrm{CL}$ 34

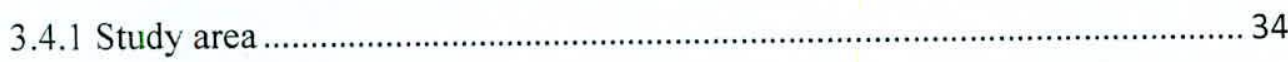

3.4.2 Collection of sand flies ......................................................................... 35

3.4.3 Sand fly identification ........................................................................... 35

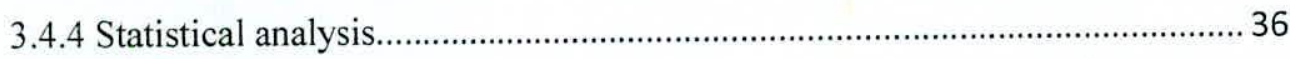

3.4.4.1 Principal component analysis (PCA) ..................................................... 37

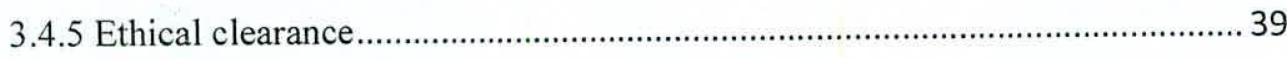

4. Results 40

4.1 First characterization of the strain causing autochthonous visceral leishmaniasis in Sri Lanka 40

4.1.1 Multi Locus Enzyme Electrophoresis (MLEE) of the VL strain. 40

4.1.2 Visceral leishmaniasis causing strain identification by 6-Phopshogluconate dehydrogenase (6PGDH) partial gene sequence and CLUSTALW alignment of partial amino acid sequence 40

4.1.3 Cutaneous leishmaniasis strain identification. 41

4.2 A cross sectional study to assess the sero- prevalence and risk factors for transmission of leishmaniasis in an endemic region of Sri Lanka 43 


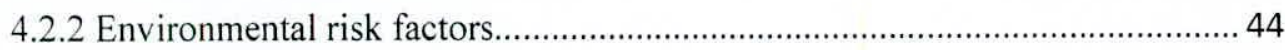

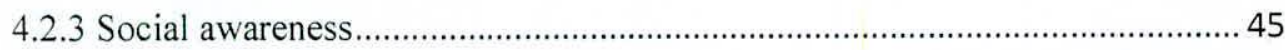

4.3 Detection of Leishmania DNA in punch biopsy samples using DNA based methods 54

4.3.1 Spectrum of clinical presentations observed during sample collection ........... 54

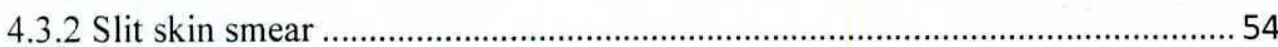

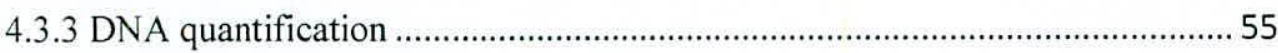

4.3.4 Sensitivity assays of the selected three sets of primers ................................ 56

4.3.4.1 Genus Leishmania specific JW11/12 primers ........................................... 56

4.3.4.2 Leishmania donovani species specific kDNA PCR (Ldf/Ldr) .................... 57

4.3.4.3 Internal Transcribed Spacer I (ITS 1) PCR (LITSR/L5.8S) ........................ 57

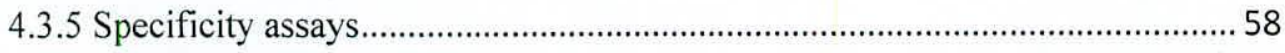

4.3.5.1 The presence of $M$. leprae DNA from a smear positive skin punch biopsy sample 58

4.3.5.2 Specificity assays of JW11/12 primers in the presence of $M$. tuberculosis, M. leprae and human DNA 60

4.3.5.3 Specificity assays of $\mathrm{Ldf} / \mathrm{Ldr}$ (L. donovani specific) primers in the presence of human, M. leprae and M. tuberculosis DNA 61

4.3.5.4 Specificity assays of LITSR/L5.8S (ITS 1) primers in the presence of human, $M$. leprae and M. tuberculosis DNA 63

4.3.6 PCR of smear positive CL punch biopsy samples......................................... 65

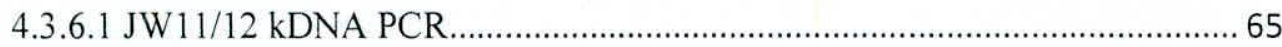


4.3.6.3 ITS 1 (LITSR/L5.8s) PCR ..................................................................... 70

4.3.7 Restriction Fragment Length Polymorphism (RFLP) …............................ 72

4.4 Taxonomic identification of sand flies captured from diagnosed CL patients' habitats

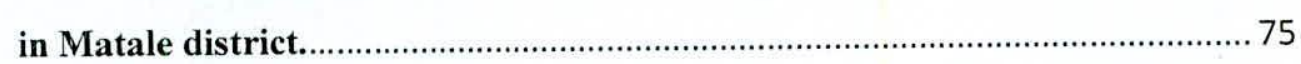

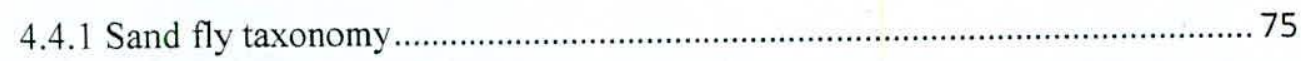

4.4.2 The male sand flies of the study population ................................................ 77

4.4.3 Principal component analysis (PCA) ……............................................... 77

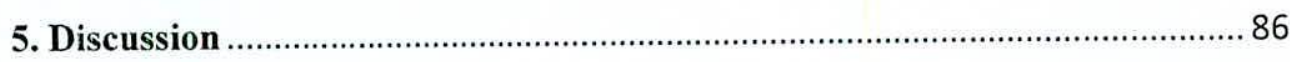

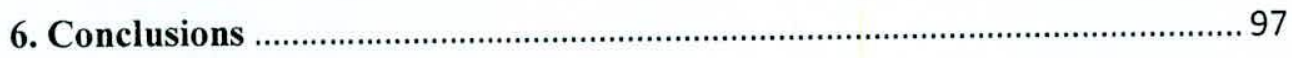

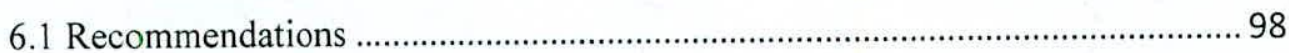

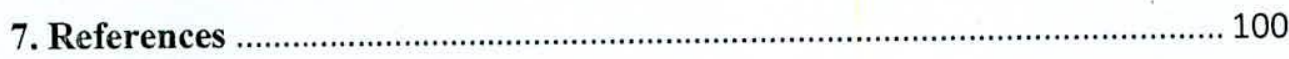

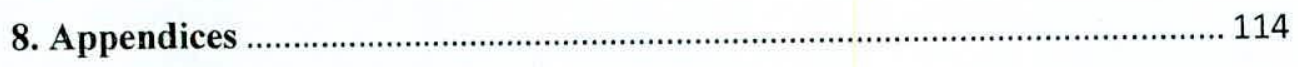

Appendix 1: List of publications and communications ..................................... 114

Appendix 2: Questionnaires \& Consent forms ................................................... 118

2.1 Title of the study: Detection of Leishmania DNA from skin biopsy samples using DNA based techniques in Sri Lankan patients 118

2.2 Title of the study: Determination of sero-prevalence in a community with active transmission of cutaneous leishmaniasis 125

2.3 Title of the study: Taxonomic identification of sand flies captured from diagnosed CL patients' habitats in Matale district. 
3.1. Title of the study: Detection of Leishmania DNA from skin biopsy samples using DNA based techniques in Sri Lankan patients. 149

3.2 Title of the study: Taxonomic identification of sand flies captured from diagnosed CL patients habitats in Matale district. 162 
Table i Distribution of the population by socio demographic characteristics and rK-39 antiLeishmania antibody detection test (RDT) status...... 48

Table ii Clinical assessment of confirmed CL patients $(n=31)$. 50

Table iii Environmental risk factors associated with $\mathrm{CL}$ 51

Table iv Summary of logistic regression analysis using CL status as the dependent variable 52

Table $\mathbf{v}$ Social awareness on leishmaniasis and the sand fly vector 53

Table vi RFLP cleavage positions 72

Table vii Summary of patients reported from different locations in the Matale district and number of identified sand flies from each location 79

Table viii Comparison of morphometric characteristics (Mean \pm SD and range) for sand flies collected at active transmission locations in the Matale district, Sri Lanka, in this study, with published data for $P$. argentipes s. $l$. 80

Table ix Component loadings for four Varimax-rotated components extracted from 11 morphological characteristics of $P$. annandalei ( $\mathrm{n}=10$; Ilango 2010), P. argentipes s.s. $(\mathrm{n}=9$; Ilango 2010), P. glaucus ( $\mathrm{n}=10$; Ilango 2010) and study sand fly population ( $\mathrm{n}=60$; Matale district). 
Figure i Autochthonous 3rd VL patient (A) and a Giemsa stained slide of the first successful invitro culture of the endogenous VL causing Leishmania strain (B). 16

Figure ii CL patient with a plaque like lesion

Figure iii Patient with a smear positive CL lesion (A), a doctor dressing up after a slit skin smear has been obtained (B) 22

Figure iv Social awareness programme and data collection at Thalawa MOH area........ 25

Figure v Partial amino acid sequence alignment of the 6-phosphogluconate dehydrogenase (6PGDH) isoenzyme from different Leishmania species. 42

Figure vi RDT results from Thalawa. 45

Figure vii RDT results from the positive control. 46

Figure viii Land use patterns of the Thalawa Medical Officer of Health $(\mathrm{MOH})$ area showing the distribution of persons screened. 47

Figure ix Spectrum of clinical presentations seen among CL patients in Sri Lanka. ......54

Figure $\mathbf{x}$ Sample 8 with very few amastigote like forms in the slit skin smear. 55

Figure xi Macrophages filled with amastigotes.. 55

Figure xii Serial dilutions of CL DNA amplified with JW11/12 primers (Rodgers et al. 1990). 56

Figure xiii Serial dilutions of CL DNA amplified with $L$. donovani specific primers (Salotra et al. 2001). 57

Figure xiv Serial dilutions of CL DNA amplified with ITS 1 primers (El Tai et al. 2001).. 58

Figure $\mathbf{x v}$ One of the smear positive lepromatous leprosy samples in acid fast stain:..... 59 
Figure xvi Mycobacerium leprae DNA amplified ( $\mathrm{n}=3 / 5$ samples) with $M$. leprae specific primers. 59

Figure xvii $M$. tuberculosis DNA amplification with JW11/12 kDNA primers. 60

Figure xviii Specificity of JW11/12 Primers in the presence of human, M. leprae and $M$. tuberculosis DNA. 61

Figure xix Specificity of $L$. donovani Ldf/Ldr primers in the presence of human $M$. leprae and $M$. tuberculosis DNA. 62

Figure $\mathbf{x x}$ M. tuberculosis DNA amplification with $L$. donovani specific kDNA primers $\mathrm{Ldf} / \mathrm{Ldr}$ (Salotra et al. 2001). 63

Figure xxi Specificity test of ITS 1 Primers (LITSR/L5.8S) in the presence of $M$. tuberculosis, $M$. leprae and human DNA. 64

Figure xxii DNA extracted from punch biopsy samples of smear positive cutaneous lesions amplified with JW11/12 primers. 66

Figure xxiii Samples that became positive with slit skin smear and negative with PCR. 66

Figure xxiv Spotting. 67

Figure xxv Spiking of PCR negative CL samples with $L$. donovni culture DNA ( $1 \mathrm{pg} / \mu \mathrm{l}) . .68$

Figure xxvi DNA extracted from punch biopsy samples of smear positive cutaneous lesions amplified with Ldf/Ldr primers. 69

Figure xxvii Nucleotide sequence from 1 to 316 of ITS 1 region. 70

Figure xxviii Testing of samples with ITS 1 (LITSR/L5.8S) primers. 71

Figure xxix Leishmania species identification by ITS 1 PCR-RFLP............................. 73

Figure $\mathbf{x x x}$ Leishmania species identification by ITS 1 PCR-RFLP. .74

Figure xxxi Matale study area. 82 
Figure xxxii Light microscope images of antennal flagellomeres, mouthparts and genitalia of male and female sand flies collected in the Matale district of Sri Lanka.. 83

Figure xxxiii Sergentomyia babu female sand fly 84

Figure xxxiv Principle component analysis regression scores for $P$. annandalei $(\mathrm{n}=10$; Ilango 2010), P. argentipes s.s. (n=9; Ilango 2010), P. glaucus $(\mathrm{n}=10$; Ilango 2010) and study sand fly population $(n=60$; Matale district $)$....... 85 


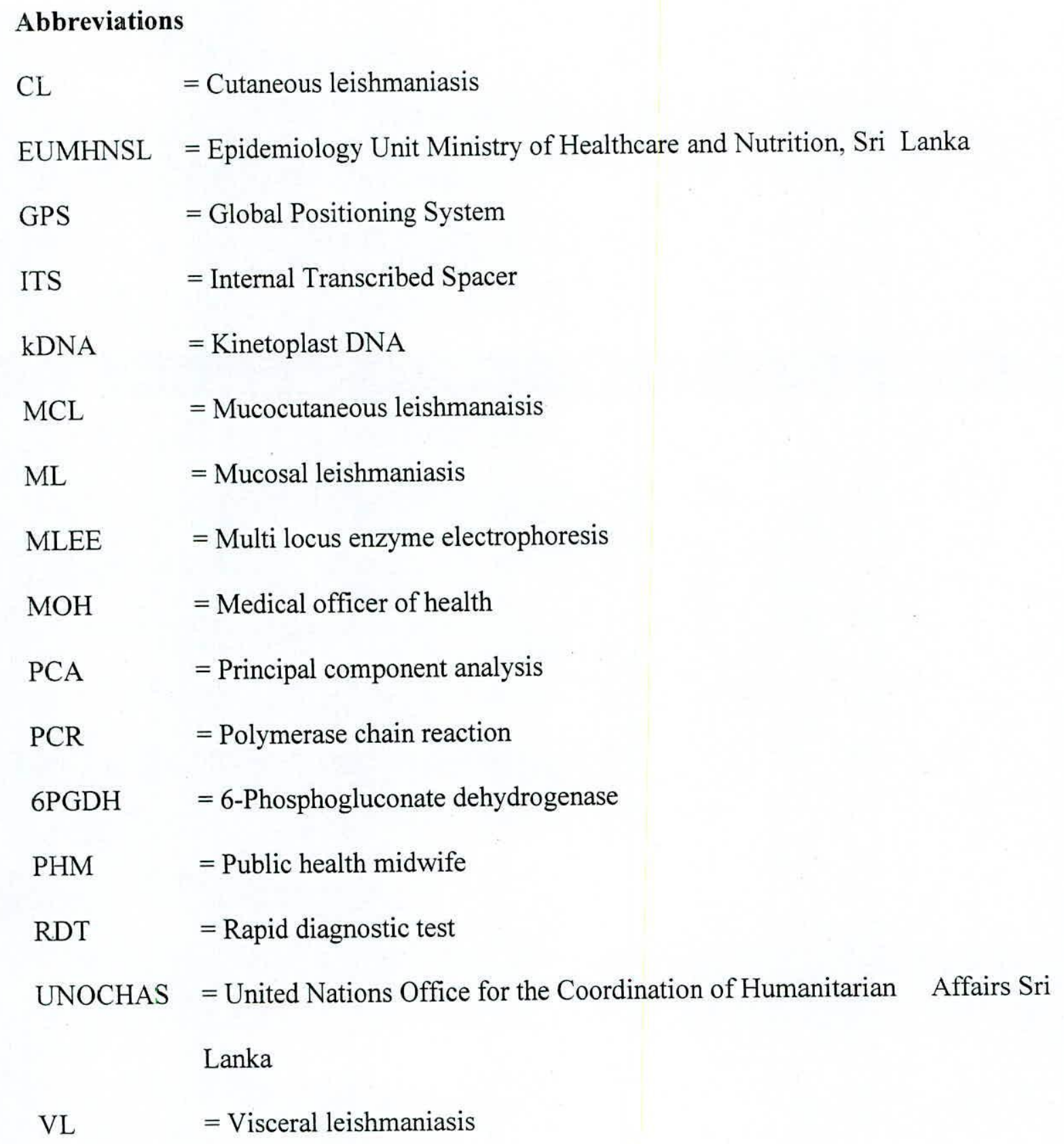




\section{Acknowledgements}

My heartiest thanks and gratitude go to my supervisors Professor Renu Wickremasinghe, Dr. NV Chandrasekharan, Dr. Rhayza Maingon, Professor Greg Matlashewski and Professor Nissanka de Silva for their kind supervision, guidance and immense help given to me in numerous ways. I thank Professor Matlashewski and Dr. Wen-wei Zhang, McGill University, Canada also for providing me with culture media, donating me 500 rK39 RDTs and carrying out the 6PGDH gene sequencing of both $\mathrm{CL}$ and VL strains isolated from Sri Lanka.

My deepest gratitude and thanks go to Dr. Francine Pratlong and Laboratoire de Parasitologie-Mycologie, CHRU de Montpellier, Centre National de Référence des Leishmania, UMR MIVEGEC (Université Montpellier 1-CNRS 5290-IRD 224), Montpellier, France, for characterization of the strain causing visceral leishmaniasis in Sri Lanka by performing by the Multi Locus Enzyme Electrophoresis (MLEE).

I would also like to thank the Consultant Dermatologists Dr. Sanjeeva Hulangamuwa, Dr. Mrs. Ganaga Sirimanna, Dr. K. Sathkurunadhan, Dr. Chandani Udagedara, Dr. Manel Dissanayake for sharing the samples and patient information with me. I thank Dr. Priyanka Abeygunasekera, Consultant Histopathologist, Dr. Sunethra Bandaranayake, Consultant Haematologist and Dr. Suresh Mendis, Consultant Physician for the deep commitment and kind collaboration given to me to for the first isolation of strain causing visceral leishmaniasis (VL) in Sri Lanka. I would also thank Dr. Asoka Munasinghe, Regional xii 
Epidemiologist, Anurahapura for helping me out with the community screening and recording of GPS coordinates in the rK39 sero-prevalence epidemiological study conducted in Thalawa $\mathrm{MOH}$ area. I would further thank Professor Rajitha Wickremasinghe, Professor Sunil Peiris and Dr. Vathsala Jayasooriya for guidance on statistical analysis, Dr. Daniel Bray for carrying out Principal Component analysis of sand fly morphometric data and Emeritus Professor Richard Ward from Keele University, UK for helping me out with the sand fly taxonomy. My sincere thanks go to $\mathrm{Mr}$. Sundaramoorthy Sivanantharajah, GIS Branch, Department of Surveys, Sri Lanka for preparing the Thalawa GPS map and calculating the distances to environmental risk factors. I would also like to thank Professor Jennifer Perera, Department of Microbiology, Faculty of Medicine, Colombo for donating me with pretested Mycobaterum tuberculosis DNA to use as a negative control. A special thank is delivered to Professor Sriyani Ekanayake for guidance given to me in various ways.

I would like to thank the staff members of the Department of Parasitology, Faculty of Medical Sciences University of Sri Jayewardenepura and Molecular biology laboratory, Department of Chemistry, University of Colombo for helping me out in numerous ways.

I would like to thank the National Research Council Grant no. 24-09 and University of Sri Jayewardenepura Research Grant no. ASP/06/RE/2008/03 for the financial assistance. I would also like to thank InBios Incorporation, Seattle, USA for the donation of $500 \mathrm{rK} 39$ rapid diagnostic kits (RDT) for my $\mathrm{PhD}$. 
Last but not the least I would like to thank my husband Harsha, two kids Anuja and Anagi and my parents for tolerating my long working hours and giving me the maximum encouragement and cooperation. 


\section{Molecular detection and sero-prevalence of Leishmania donovani \& risk factor analysis in selected areas in Sri Lanka.}

\section{Peduru Hewa Kankanange Imalka Shalindra Ranasinghe}

\section{ABSTRACT}

Introduction: Leishmaniasis is a newly emerged and established disease in Sri Lanka. This study describes the application of a highly sensitive and specific Leishmania DNA based assay to skin punch biopsy samples, determination of sero-prevalence of antiLeishmania antibodies, risk factor analysis for transmission of leishmaniasis in an 'at risk' community and sand fly identification by traditional methods in diagnosed patients' habitats.

Methodology: The Leishmania strain causing visceral leishmaniasis (VL) in Sri Lanka was characterized by Multi Locus Enzyme Electrophoresis and VL and cutaneous leishmaniasis (CL) causing strains were confirmed by sequencing the 6-phosphogluconate dehydrogenase (6PGDH) gene. Three sets of previously described primers (kDNA: JW11/12, Ldf/Ldr and ITS 1:LITSR/L5.8S) were used to detect Leishmania DNA in skin punch biopsy samples. Digestion of the ITS 1 region with Hae 111 and Restriction Fragment Length Polymorphism (RFLP) was used for parasite identification.

A cross sectional study of 1000 individuals, was carried out in a high CL prevalent area, \{estimated population $\sim 61,674$, Thalawa Medical Officer of Health $(\mathrm{MOH})$ area\}, to assess presence of the rK39 anti-Leishmania antibody in sera, (with the rK39 rapid diagnostic 
test, RDT); CL prevalence with microscopy of slit skin smears, and to analyze risk factors for transmission of leishmaniasis using a pre-tested, interviewer administered, questionnaire and global positioning system. Randomly selected sand flies ( $\mathrm{n}=96$ ) from habitats close to diagnosed CL patients houses were captured using the cattle bait from Matale district, and taxonomically identified using taxonomic keys. Morphometric measurements were analyzed and compared with previously described data using the student's $t$-test and principal component analysis (PCA).

Results: The endogenous VL causing strain in Sri Lanka was identified as Leishmania donovani MON-37 by MLEE. The CL strain isolated was also characterized as Leishmania donovani MON-37 by sequencing the $6 \mathrm{PGDH}$ gene. The sensitivity of JW and ITS 1 primers in detecting Leishmania DNA by PCR was $92 \%(n=35 / 38)$. Sensitivity assay using Ld primers by PCR was $71 \%(\mathrm{n}=27 / 38)$. The specificity of JW and ITS 1 primers were $100 \%$ while $\mathrm{Ld}$ primers showed some cross-amplification with Mycobacterium tuberculosis DNA. All amplified ITS 1 fragments following digestion with Hae 111 RFLP gave a pattern characteristic of $L$. donovani. The prevalence of $C L$ was $3.2 \%$ (31/954) and sero-positivity to anti-Leishmania antibodies was $0.1 \%$ in Thalawa $\mathrm{MOH}$ area. Living in close proximity to paddy fields has shown to be the only risk factor ( $\mathrm{p}=0.01$ ) for the transmission of leishmaniasis in Thalawa. Out of the 96 sand flies identified 95 were $P$. argentipes and one was genus Sergentomyia. PCA analysis showed that $P$. argentipes identified from Sri Lanka formed a separate cluster when compared with the recently described $P$. argentipes, $P$. annadale $i$ and $P$. glaucus sibling species indicating existence of a morphologically distinct of $P$. argentipes population in Sri Lanka. 
Conclusions and recommendation: L. donovani MON-37 causes both $\mathrm{CL}$ and VL in Sri Lanka. JW11/12 and ITS 1 primers could be recommended to detect Leishmania DNA in skin biopsy samples from Sri Lankan patients. High prevalence of CL was detected in the study area and $P$. argentipes in Sri Lanka form a separate morphological cluster. These results would indicate the need for comparison studies to detect phenotypic and genotypic differences in CL and VL causing Sri Lankan L. donovani MON 37 strains, need for active case detection studies, improve surveillance of $\mathrm{CL}$, early treatment to reduce the human reservoir, studies on sand fly biology and implementation of control measures to control and combat the spread of leishmaniasis in the country 